Session 2620

\title{
BattleBots and the Electrical Engineering Education
}

\author{
Barry E. Mullins, Brian S. Peterson \\ Department of Electrical Engineering / Air Force Institute of Technology \\ United States Air Force Academy, CO / Wright-Patterson Air Force Base, OH
}

\begin{abstract}
The use of robotics as a learning tool within computer/electrical engineering as well as computer science curriculums is ever increasing for a variety of reasons including stimulating interest in engineering. This paper describes the educational experiences gained through the design, construction, and competition of two robots called BattleBots. A television show sponsored by BattleBots Inc. showcases these BattleBots in a radio-controlled robotic combat competition. The show is televised by Comedy Central within the United States. Competitors design, build, and test a fortified robot in hopes of attending the biannual, single-elimination tournament, incapacitating the competition, and walking away with the top prize. During the spring of 2001, two electrical engineering students at the United States Air Force Academy (USAFA) built two robots as their capstone senior design projects and subsequently competed in the May 2001 tournament. This paper describes the background of BattleBots, the process by which the students completed their robots, how the students were able to attend the competition, and how the students faired in battle. In addition, the tremendous national media coverage of the students, and the ramifications of the students attending the contest are discussed. The paper points out the enthusiasm of the students as they built their robots and prepared for the competition. It is shown that using BattleBots as a motivational venue exposes the general public and students to the advantages of pursuing an engineering degree.
\end{abstract}

Introduction

It is well documented that the use of robots stimulates the learning process in an educational environment. One of the ancillary benefits of using robots in the classroom or lab is that it forces the students to consider cross-disciplinary issues. Systems engineering takes on a whole new meaning - students must address the interfaces between digital, analog, and mechanical systems. It is no coincidence these issues are addressed by ABET. ABET now requires curricula to include cross-disciplinary, project-oriented learning. The use of robots in our curriculum satisfies these requirements. In addition, these issues satisfy the critical educational outcomes of USAFA. Moreover, building a BattleBot satisfies the goal of our senior capstone-design course (EE 464). The goal of EE 464 "is for all electrical engineering cadets to gain practical experience in the 'real world' of engineering problem solving by the successful design and implementation of a challenging electrical engineering project" . 
Ideas for EE 464 projects originate from numerous avenues: professor interests, student suggestions, requests from outsides sources, or satisfying Air Force needs. The BattleBot project was suggested by an eager student who had seen the television show and was interested in building his own BattleBot. Numerous students at USAFA (including non-EE majors and undeclared majors) as well as their parents had seen the show and the thought of building and competing was exciting. The thrill of competition is usually a good motivator, and the possibility of appearing on national TV was even more incentive to excel. His request was considered by the faculty. It isn't often a student displays the degree of enthusiasm we saw in our BattleBot students. Based on engineering merit and student enthusiasm, the project was deemed as a worthy capstone project with stipulations: the students were required to design a major subsystem from scratch.

Most BattleBot builders buy all of the major subsystems prefabricated (speed controller, radio control transmitter/receiver, motors) and then assemble them to form a complete, operational robot. That alone would not be a worthy challenge for a senior electrical engineering student. It was assumed that the student would choose the speed controller and/or radio control system to build, since actual motor design and fabrication has very low tolerance for variation.

The date of the official competition was unknown at the onset of these projects, so it was recommended that at least two students build BattleBots so a local competition could be held if the May 2001 competition could not be attended. The faculty thought a BattleBots competition, local or nationally televised, would be an excellent venue for grabbing the attention and interest of undeclared students and possibly directing them toward engineering. Moreover, if the students went to the televised competition, it would be great publicity for the institution and for the Air Force. Fortunately, another student did step forward to build a second BattleBot.

\section{BattleBot Robots}

The students were required to build the robots following BattleBots competition specifications. The rules were quite extensive, but essentially contained the following. Each robot fell into a competition weight class: lightweight, middleweight, heavyweight, or super heavyweight. The robot could be powered electrically, hydraulically, pneumatically, mechanically, or by internal combustion engine with a limited amount of fuel. Each robot had to be controlled by radio frequency remote control using unlicensed bands or licensed bands if the proper permissions are secured. Pulse Code Modulated (PCM) radio systems were recommended. No untethered projectiles (including flammable liquids), electromagnetic pulses, or high power lasers were permitted. The robots could be wheeled, walk, hop, or even hover.

The competition consists of robots dueling in single elimination one-on-one battles in the 'BattleBox', which is a square 48-foot arena with clear one-inch thick Lexan walls and is filled with destructive obstacles the competitors must avoid. Such obstacles include 'kill saws', which are high power saws that rise out of the arena floor, and the 'pulverizers' - large aluminum mallets which slam down to smash competitors. Each battle lasts three minutes. The winner is determined when the opponent's robot is incapacitated or by judges' decision. The ultimate goal is to demolish the opponent robot in dramatic fashion with its components flying all over the arena. 
Each robot has five major components: chassis, power system, remote control system, direction controller, and speed controller. The chassis includes the mechanical frame and the weapons (if any). The power system provides mobility, drives the weapons, and provides power for the electronic control system. The remote control system consists of a transmitter/receiver pair for the radio link between the robot and the operator(s) outside of the BattleBox. The controller takes the signals from the receiver and translates them into a format the motors can use. The speed controller then amplifies the current of the signals to actually drive the motors. Each robot is different and may have multiple weapons, multiple power systems, and even multiple radio links.

\section{EE 464 Restrictions}

As mentioned earlier, the students were expected to design, build, and test one of the electrical subsystems from scratch. Since the robot design is very dependent on the chassis and weapons system, the students also had to design the mechanical portions of the robot. They could then use a staffed fabrication facility at USAFA to assist them in the construction of the robot chassis. Nominally, each project is provided a budget of a few hundred dollars for parts and materials.

Each student met with the instructors/mentors to review his design and progress at least once a week. The instructor could take this time to gently nudge the student back onto a proper engineering design path in case he began to go astray. Since this was an opportunity for learning, the instructor typically questioned the students' methods to make sure he had a sound plan and rarely intervened in the design process unless it was obvious that the student was heading in a direction that would doom the project to failure. Inexpensive mistakes were allowed and expected as part of developing into a qualified engineer.

\section{The Designs}

Both students chose to build middleweight robots (59-116 lbs) in order to maximize their flexibility in structure and weapons without the robot becoming too unwieldy to handle. Both students also chose to purchase motors/engines and transmitter/receiver pairs and design the controller and speed controller (since they both had considerable experience with microcontrollers and transistor technology). Speed controller design, however, is not specifically taught in the EE curriculum at USAFA so the students had to research and learn about speed controller design on their own. Many BattleBot builders purchased pre-programmed controllers and most buy commercial speed controllers.

\section{‘Mordicus’ Built By Jon}

The first student, Jon, set out with the goal of making the strongest, highest power robot that the budget and rules would allow. His goal was to destroy anything that came within reach of his robot. He contacted veteran competitors for tips, did considerable research into all aspects of his robot, and pushed his designs to the limit. He researched metals to determine the strongest, yet most affordable metals to use. He researched several types of weapons and decided on two metal cutting blades placed in a manner that would draw the victim into both blades to allow for the longest cut time possible. His design resulted in a two-wheeled robot with a wedge at one 
end and the blades at the other. The chassis was made of aluminum that was to be covered with a layer of tempered spring steel. Jon named his robot Mordicus (Latin for "teeth"). Figure 1 illustrates the configuration of Mordicus.

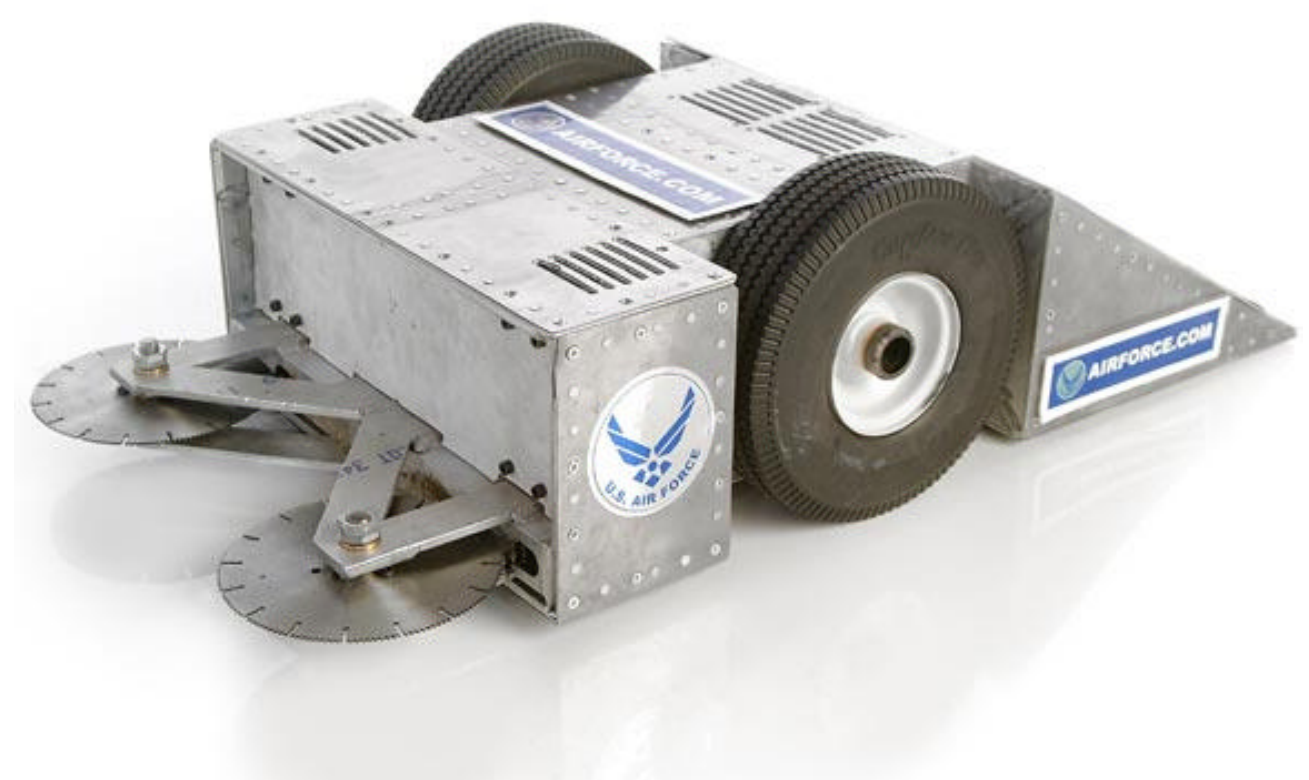

Figure 1. Mordicus

(Photo courtesy of BattleBots Inc., Copyright 2001 BattleBots Inc., Daniel Longmire, Photographer)

Jon performed extensive research to find the smallest motors that could deliver the most torque. His system was controlled using the Motorola $68 \mathrm{HC} 11$, which he had used in several courses. Designing the detailed interface hardware and the software for the microcontroller included conditioning the signals so they could be read into the $68 \mathrm{HC} 11$ from the transmitter/receiver pair and coding the $68 \mathrm{HC} 11$ to output the correct signals to the speed controller circuitry for the motors. This was time consuming, but gave him few major problems. The problems came in designing the speed controller.

Due to his high performance parts (e.g., motors) and expectations of his system, the speed controller was required to amplify high current loads very quickly. The MOSFET transistors required to do this are relatively expensive. Jon continued his trend of trying to make his system as elegant as possible and tried to drive a motor controller that usually needs $24 \mathrm{~V}$ with just $12 \mathrm{~V}$ (to minimize battery size and weight) with novel, non-standard technology. This generated problems which began to lead him down a path away from solid design methods to juryrigging' it to fix the problems as they popped up. The instructor advised him to review and take a more disciplined design approach. He then decided to cut his losses on his original approach and found a simple, more stable solution.

Jon decided that, based on performance and thermal protection requirements, MOSFET transistors exceeded his budget. Unfortunately, he destroyed several of them in the process of debugging his H-bridge (speed controller configuration) that provided the current amplification. 
The system would periodically encounter 'shoot-through' which resulted in transistors overheating to the point of flames shooting out of the circuitry. In the end, he was eventually able to eliminate most of the 'shoot-through' problems with further careful engineering, but not all.

\section{“Something Must Die (SMD)" Built By Danial}

The second student, Danial, took a more relaxed approach and endeavored to build a simpler robot that could at least hold its own in the arena. His preliminary design included a chainsaw engine driving a spinning steel disc and propelled by two wheels attached to DC motors. Figure 2 shows SMD. The electronics would receive signals from the receiver and translate them into motor movements, as well as control a throttle servo on the chain-saw motor. He decided that instantaneous acceleration and high speed were not necessary and, therefore, chose medium performance motors. As a result, he was able to choose thermally protected MOSFETS that would turn off temporarily in the event of overheating. Thus, he did not run into the same 'shoot-through' glitches that plagued Jon.

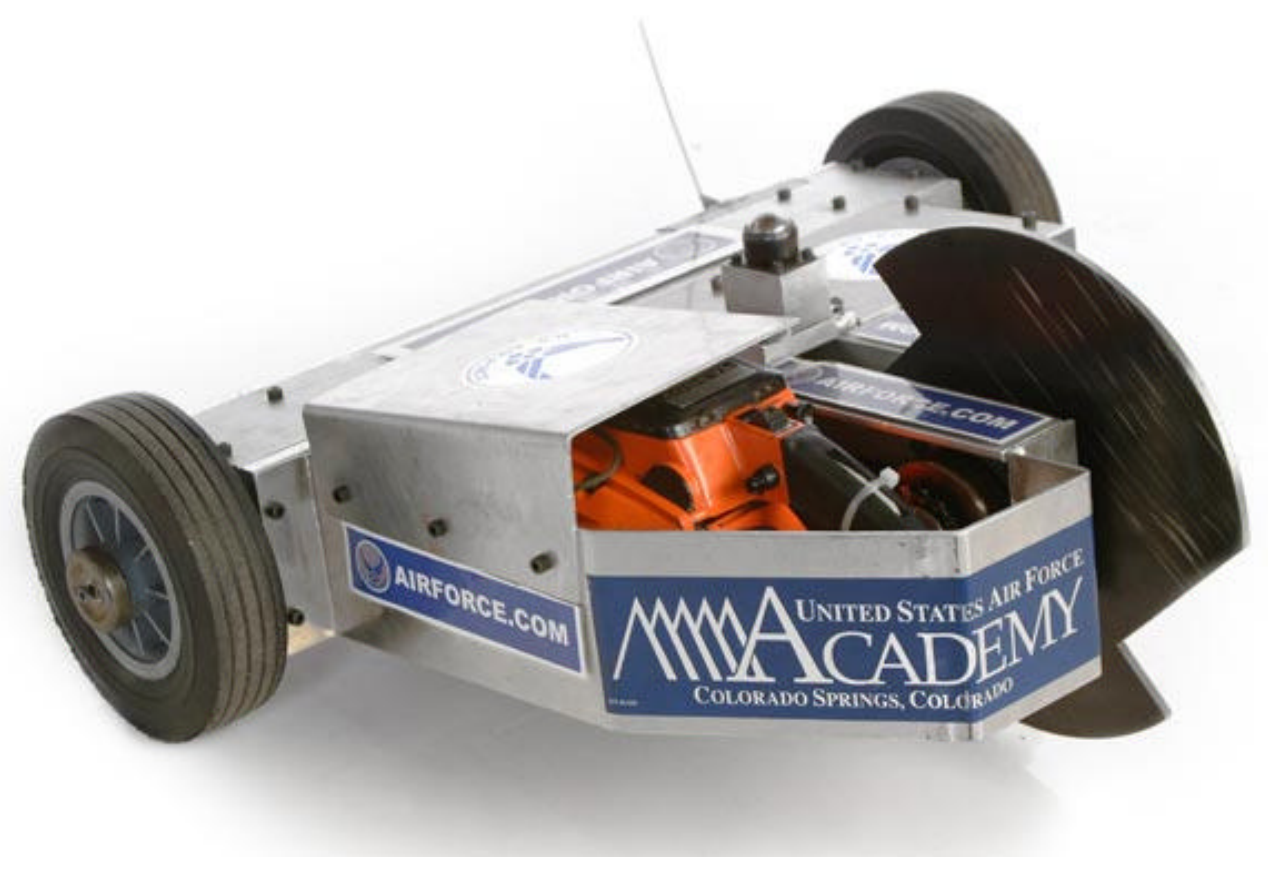

Figure 2. Something Must Die

(Photo courtesy of BattleBots Inc., Copyright 2001 BattleBots Inc.,

Daniel Longmire, Photographer)

Danial chose to use a Prototype Intelligent Controller (PIC) due to the low cost of each chip and for the general learning experience. Since specific PICS are not covered in the EE curriculum, this involved research in order to control the PIC rather than the more complex 68HC11: its capabilities, its language, and the actual programming process. Danial's development went relatively smoothly, though it involved a considerable time investment. He used some of Jon's research on $\mathrm{H}$-bridge design, which made his task easier. 
Danial's primary problems arose from the electromagnetic interference (EMI) from the spark plug on the chain-saw engine. When the spark plug fired, it would translate a pulse onto the PIC electronics and disrupt its operation. After some shielding and changes in coding, that problem was apparently fixed at USAFA before leaving for the competition.

Mechanical Fabrication

These projects were more involved than most EE 464 projects due to their mechanical aspects. As mentioned, the students were required to complete the design and much of the mechanical fabrication on their own. This consumed a lot of their time and pushed the scope of the project beyond what is normally expected of EE 464 projects. Still, the students learned a great deal about mechanical design during the process.

Pre-competition

Designing, fabricating, and testing the BattleBots was half the challenge. Attending the competition remained the ultimate goal for the students as well as the faculty mentors. This entailed a series of nontrivial tasks including: obtaining approval from the Air Force including the USAFA chain of command and legal office, coordinating efforts with Air Force public affairs officials, coordinating the trip to the competition with the Air Force Recruit ing Service, and securing funding.

Since USAFA is a military organization, obtaining approval to represent the Air Force and USAFA was absolutely essential. Ultimately, approval from the highest-ranking officer at USAFA - the superintendent (a three-star general) — was required. The students and faculty mentors were delighted to discover that everyone involved in the decision process wholeheartedly supported the request. Some officers had actually seen the BattleBots show and saw the opportunity as an excellent venue to highlight USAFA on a national scale. Everyone saw the opportunity as a means to underscore the prowess of USAFA's engineering program.

Since the students were working on the BattleBots right up to the competition date, the faculty decided to package everything (BattleBots, spares, equipment, etc.) in shipping containers and check them on the commercial flight to Oakland California. Seven containers ranging in weight from 75 to 100 pounds were required to comply with the weight and size limitations of the airline. Naturally, the airline assessed fees for checking overweight and oversized luggage.

\section{At The Competition}

The May 2001 BattleBots competition was held in a vacant hanger on Treasure Island near San Francisco CA. This venue provided everyone with space to prepare his or her BattleBot(s) for competition. After registration, both students readied their robots for the first round in this single elimination tournament. Both BattleBots were functional, yet could be improved. Jon continued to work on his drive electronics problems in Mordicus; Danial worked on the EM interference from the chainsaw engine, which reappeared when the robot was powered in California. Each student spent the evenings either fixing problems or fine-tuning their BattleBots. Both students demonstrated outstanding engineering skills by improvising when 
confronted with problems not solvable with the spare parts and equipment taken to the competition.

Danial's first match was against a titanium spinner with a very low profile. Since he would never be able to get his spinning disk under the spinner, he decided not use the chainsaw motor at all. He was able to defeat the spinner by simply letting his opponent spin up and then ramming it. Eventually, the spinner developed an electronics problem due to a connector shaken loose by multiple impacts. With one win under his belt, Danial set out to finally solve his EM interference problem. Using his knowledge of the interference and his microcontroller's capabilities, he devised a software solution by writing a low pass filtering algorithm for his controller. The algorithm was designed to negate the impulses generated by the spark plug on the chainsaw. His solution work flawlessly.

His second match was against another titanium robot. During the course of the match, his robot was flipped. Usually, this isn't a problem for SMD; however, his antenna was now being dragged behind him and his opponent ran over it grounding it to the metal flooring. Regrettably, Danial lost radio contact with SMD and was incapacitated. The bout then ended - being considered a knockout.

Jon's first match was a true test of his BattleBot Mordicus. His opponent was another titanium BattleBot in the shape of an inverted boat hull. Although he put up a good fight, one side of his drive system went out leaving Mordicus with the ability to spin only. His opponent was able to get in a few good hits and was subsequently declared the winner by the judges in a close decision.

BattleBots is televised on the Comedy Central network. To make the shows more interesting and provide a glimpse into a BattleBot builder's life, Comedy Central often profiles builders. Profiling simply involves filming the builder going about his or her everyday life while building the BattleBot. Comedy Central and BattleBots had selected our team to profile during this season. In order to make Comedy Central's visit to USAFA meaningful, they needed some footage of the two BattleBots in action at the competition. Since both students lost in early rounds, they were not videotaped; only later matches were actually taped. As it turns out, several of the teams scheduled to be profiled did not advance to a televised round. Therefore, BattleBots arranged for an exhibition robot rumble pitting "The Brightest Minds of the Nation" against Jay Leno's (host of NBC's “The Tonight Show with Jay Leno") massive 450-pound BattleBot called Chin-Killa. This match was videotaped and later televised. Figure 3 is an image captured from the televised show. This particular scene was during the profile of the students.

The experience at the competition was amazing. The students met several great engineers ranging from student to seasoned veterans. Everyone exhibited tremendous sportsmanship. All competitors were enthusiastic and willing to help each other. 


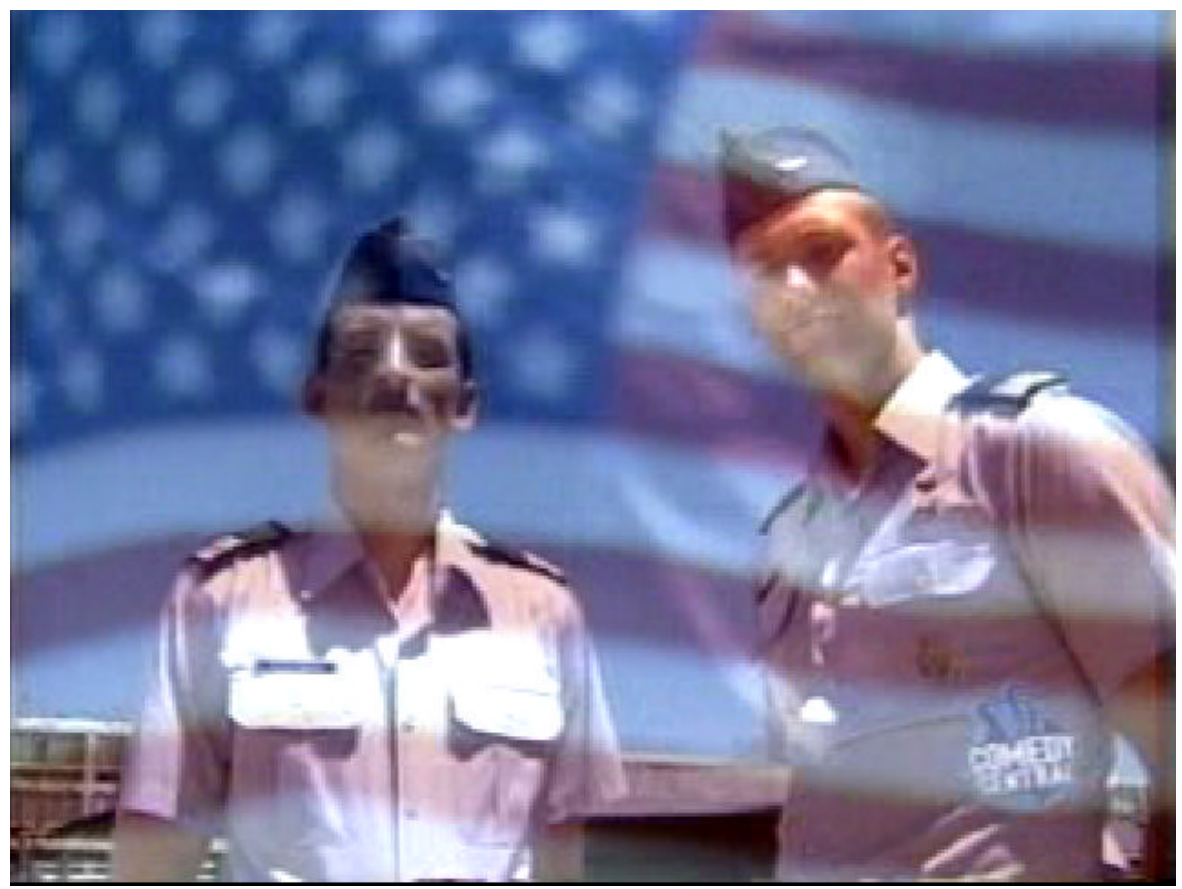

Figure 3. Image of Danial and Jon from Televised BattleBots Show

\section{Media Coverage}

Beyond the actual televised match against Chin-Killa, the media coverage of the students was incredible. The students were also interviewed by Newsweek magazine and highlighted in the 28 May 2001 issue $^{2}$. Additionally, they were showcased in the base (campus) newspaper, Academy Spirit $^{3}$, Air Force News ${ }^{4}$, Air Force Radio ${ }^{5}$, the USAFA Association of Graduates publication Checkpoints $^{6}$, and local San Francisco television stations. Mordicus ${ }^{7}$ and SMD $^{8}$ as well as the other teams can be found on the BattleBots webpage.

As a result of the media exposure, the students have been invited to technology fairs in nearby towns to expose other students at other universities to engineering applications. Moreover, several undeclared students have expressed an interest in an electrical engineering degree after seeing the show and seeing the BattleBots at open houses. The faculty members are also being sought by other universities to provide advice on how to compete as well as the logistics involved.

\section{Conclusions}

BattleBots are outstanding projects for capstone design courses. Student enthusiasm is the highest seen in several years. The students knew they were representing the Air Force and USAFA; therefore, they devoted themselves completely to the projects. The level of project difficulty appeared appropriate. The students were challenged both from an electrical/computer engineering perspective as well as mechanical perspective. 
This was the first year for USAFA's involvement in BattleBots. Based on the exceptional experience, the department anticipates participating in BattleBots for several years to come. It is envisioned that the BattleBots will grow in sophistication as subsequent students attempt to build their version of a BattleBot. As they do, the faculty mentors also gain a better appreciation for power electronics as applied to high-powered mobile robots.

\section{References}

1. EE 464 Course Assessment Plan Version 1.1, United States Air Force Academy CO, 31 January 2000.

2. Stone, Brad, "Attack of the BattleBots", Newsweek, pgs. 40-42, 28 May 2001.

3. Arredondo, Angela, "Robotic Warriors Go National with their BattleBots", Academy Spirit, pg. 12, 18 May 2001.

4. URL: http://www.af.mil/news/May2001/n20010522_0693.shtml

5. URL: http://www.af.mil/news/radio/May2001/20010524.ram

6. Arredondo, Angela, “Getting It (Just) Right”, Checkpoints, pgs. 32-33, Summer 2001.

7. URL: http://www.battlebots.com/meet_the_robots/meet_robot_specs.asp?id=493

8. URL: http://www.battlebots.com/meet_the_robots/meet_robot_specs.asp?id=490

BARRY E. MULLINS is an Assistant Professor and Chief of the Computer Systems Division within the Department of Electrical Engineering at the U.S. Air Force Academy. He received a B.S. in Computer Engineering from the University of Evansville, a M.S. in Computer Engineering from the Air Force Institute of Technology, and a Ph.D. in Electrical Engineering from Virginia Tech. Lt Colonel Mullins is a member of ASEE, IEEE (Senior Member), Tau Beta Pi, and Eta Kappa Nu. His research interests include mobile robots, microprocessor/ microcontroller-based systems and applications, and wireless local area networks. Email: barry.mullins@usafa.edu

BRIAN S. PETERSON is a Ph.D. student at the Air Force Institute of Technology. At the time of the BattleBots project, he was an Assistant Professor in the Computer Systems Division within the Department of Electrical Engineering at the U.S. Air Force Academy. He received a B.S in Electrical Engineering from the U.S. Air Force Academy, a M.S. in Systems Engineering from AFIT and a M.S. in Electrical Engineering from Florida State University. Capt Peterson is a member of ASEE, IEEE, and Tau Beta Pi. Email: brian.peterson@afit.edu 